\title{
Preparation and Characterization of Water-Soluble Chitosan Microparticles Loaded with Insulin Using the Polyelectrolyte Complexation Method
}

\author{
Sihui Wu, ${ }^{1}$ Yi Tao, ${ }^{2}$ Hongliang Zhang, ${ }^{2}$ and Zhengquan Su${ }^{3}$ \\ ${ }^{1}$ Department of Pharmacy, Guangdong Food and Drug Vocational-Technical School, Guangzhou 510663, China \\ ${ }^{2}$ College of Pharmacy, Guangdong Pharmaceutical University, Guangzhou 510006, China \\ ${ }^{3}$ College of Public Health, Guangdong Pharmaceutical University, Guangzhou 510006, China
}

Correspondence should be addressed to Zhengquan Su, suzhq@scnu.edu.cn

Received 26 July 2010; Revised 17 November 2010; Accepted 7 January 2011

Academic Editor: Maryam Tabrizian

Copyright (C) 2011 Sihui Wu et al. This is an open access article distributed under the Creative Commons Attribution License, which permits unrestricted use, distribution, and reproduction in any medium, provided the original work is properly cited.

\begin{abstract}
Polymeric delivery systems based on microparticles have emerged as a promising approach for peroral insulin delivery. The amount of insulin was quantified by the improved Bradford method. It was shown that water-soluble chitosan/insulin/tripolyphosphate (TPP) mass ratio played an important role in microparticles formation. Stable, uniform, and spherical water-soluble chitosan microparticles (WSC-MPs) with high insulin association efficiency were formed at or close to optimized WSC/insulin/TPP mass ratio. WSC-MPs had higher association efficiency in the $\mathrm{pH} 4.0$ and $\mathrm{pH} 9.7$ of TPP solution. The results showed that association efficiency and loading capacity of insulin-loaded WSC-MPs prepared in $0.01 \mathrm{~mol} / \mathrm{L} \mathrm{HCl}$ of insulin were $48.28 \pm 0.90 \%$ and 9.52 $\pm 1.34 \%$. The average size of insulin-loaded WSC-MPs was $292 \mathrm{~nm}$. The presented WSC microparticulate system has promising properties towards the development of an oral delivery system for insulin.
\end{abstract}

\section{Introduction}

In the present paper, insulin was chosen as the model protein. It is a well-known 51 amino acids protein, and the oral approach remains the most attractive due to convenience and high patient compliance [1]. However, administering drugs orally is by far the most widely used route of administration, although it is generally not feasible for protein drugs. The main reasons for the low oral bioavailability of biologicals are presystemic enzymatic degradation and poor penetration of the intestinal membrane [2,3]. Much has been learned in the past few decades about macromolecular drug absorption from the gastrointestinal (GI) tract, including the barriers that restrict GI absorption [4]. One approach to improve the gastrointestinal uptake of low molecular weight proteins is to bind them to colloidal systems like microparticles (MPs), protecting them from degradation in the gastrointestinal tract and promoting the transport into systemic circulation [5-8].
MPs are defined as particulate dispersions or solid particles with a size in the range of $100-1000 \mathrm{~nm}$. The drug is dissolved, entrapped, encapsulated, or attached to a micro-particle matrix. The major goals in designing microparticles as a delivery system are to control particle size, surface properties, and release of pharmacologically active agents in order to achieve the site-specific action of the drug at the therapeutically optimal rate and dose regimen [9]. More recently, researches have attempted to study chitosan microparticles (CS-MPs) as follows: preparation, modification, properties of loading various drugs, and their physiological characters, such as CS-MPs coated PLGA [10] and PEG [11], MPs loaded insulin [12], DNA [13], vaccine [14], peptide [15], enzyme [16], and anticancer drug 5fluorouracil [17]. But some important factors affecting drug properties have not always been investigated, such as the type of water-soluble chitosan (WSC), and were seldom evaluated in insulin delivery system of nanoparticles [18]. Polyethylene glycol (PEG-) coated nanoparticles have been 
found to be important potential therapeutic application for controlled release of drugs and site-specific drug delivery [19]. Therefore, we investigated a series of factors affecting delivery properties, concentration of WSC, and initial insulin which were all evaluated.

The objective of the present study was to evaluate the effects of formulation parameters on the insulin association efficiency of the insulin-loaded WSC-MPs and determine the effects of insulin association efficiency on different $\mathrm{pH}$ of TPP and resolution medium of insulin and release properties during incubation in phosphate buffer saline (PBS) of pH 7.4 and $\mathrm{pH} 1.2$ and investigate the physicochemical structure of insulin-loaded WSC-MPs by TEM and particle size analyzer. A series of insulin-loaded WSC-MPs with various parameters were prepared. The physical and morphological properties of insulin-loaded WSC-MPs were investigated in accordance with formulation parameters, and the release profile of insulin was also determined regarding its potential for oral delivery.

\section{Materials and Methods}

2.1. Materials. WSC (degree of deacetylation $87 \%$, molecular weight $21 \mathrm{kDa}$ ) was obtained from Shandong AK Biotech Ltd. Porcine insulin $\left(27.8 \mathrm{IU} \cdot \mathrm{mg}^{-1}\right)$ was purchased from Xuzhou Biochemical Plant, China. TPP was purchased from Tianjin Hongyan Chemical Reagent Factory. Coomassie Brilliant Blue G-250 was obtained from MBCHEM. All other chemicals and solvents were of analytical grade.

2.2. Equipment. UV-2201 UV-analyzer (Shimadzu, Japan), JB-3 Magnetic stirrer (Shanghai Instrument, China), SORVALL Biofuge Stratos low-temperature high-speed centrifuge (Thermo, Germany), THZ-051 high-precision CNC shaker (Thermo, Germany), ALPHA1-4 LSC Freeze Dryer (Martin Christ, Germany), Zetasizer 3000 HAS (Malvern, UK), TEM-100CXII (Electronics Company, Japan).

2.3. Preparation of Chitosan Microparticles. WSC-MPs were prepared by the ionic gelation of WSC and tripolyphosphate (TPP) as described by Fernández-Urrusuno [20]. Briefly, WSC was dissolved in aqueous solution in various concentrations. Then, tripolyphosphate (TPP) was dissolved in distilled water. Microparticles were spontaneously obtained upon the addition of $1 \mathrm{~mL}$ of a TPP aqueous solution to $5 \mathrm{~mL}$ of the WSC aqueous solution under magnetic stirring at room temperature. The resultant mixtures were broadly characterized as either a clear solution, an opalescent suspension (MPs), or aggregates. The optimal solution concentrations were identified from these experiments as $0.8-3.0 \mathrm{mg} / \mathrm{mL}$ WSC in aqueous solution and $0.5-2.0 \mathrm{mg} / \mathrm{mL}$ TPP in distilled water. These concentrations were subsequently used for the preparation of micro-particle suspensions.

2.4. Preparation of Insulin-Loaded WSC-MPs. Insulin-loaded WSC-MPs were prepared by the polyelectrolyte complexation of WSC with insulin and TPP as a counteranion, in which the positively charged amino groups of WSC interact with the negatively charged TPP [21]. Water-soluble chitosan $(1.0-2.0 \mathrm{mg} / \mathrm{mL})$ as well as TPP $(0.7-1.5 \mathrm{mg} / \mathrm{mL})$ were dissolved in distilled water. Insulin was dissolved in $0.01 \mathrm{M} \mathrm{HCl}$ at various concentrations. Insulin solution $(2 \mathrm{~mL}$ ) was premixed with $2 \mathrm{~mL}$ of TPP solution or $4 \mathrm{~mL}$ of WSC solution before the addition of the TPP solution dropwise into the WSC solution under magnetic stirring at ambient temperature. Insulin-loaded WSC-MPs were isolated by centrifugation $\left(15,000 \times \mathrm{g}, 30\right.$ minutes, $\left.4^{\circ} \mathrm{C}\right)$ and resuspended in $10 \mathrm{~mL}$ of purified water after discarding the supernatants. Insulin-loaded WSC-MPs were also prepared on a large scale by adding $20 \mathrm{~mL}$ of the TPP solution and $20 \mathrm{~mL}$ of the insulin solution to $40 \mathrm{~mL}$ of WSC, maintaining the stirring conditions (at room temperature). Insulinloaded WSC-MPs were centrifuged $(15,000 \times \mathrm{g}, 30$ minutes, $4{ }^{\circ} \mathrm{C}$ ), and the resuspension volumes were proportionally adapted to. Freeze-drying was performed using the freeze dryer. Particles were dried for $24 \mathrm{~h}$ corresponding to a temperature of $-45^{\circ} \mathrm{C}$.

2.5. Micro-Particle Characterization. The particle size and size distributions of the insulin-loaded WSC-MPs were determined by particle sizer (Zetasizer 3000 HAS, Malvern Instruments Ltd., Worcs, UK). Insulin-loaded WSC-MPs separated from suspension were dried by a freeze dryer. The morphology of the MPs was observed by TEM-100CXII.

2.6. Insulin Association Efficiency. The association efficiency (AE) and loading capacity (LC) of the process were determined upon separation of the insulin-loaded WSC-MPs by ultracentrifugation at $15,000 \times \mathrm{g}, 4^{\circ} \mathrm{C}$ for 30 minutes from the aqueous medium containing nonassociated insulin. The amount of free insulin in the supernatant was measured using the improved Bradford method [22]. The $\mathrm{pH}$ of supernatant of insulin-loaded WSC-MPs were adjusted to 7-9 using saturated $\mathrm{NaOH}$, and the precipitations (free chitosan) were separated from solution by centrifuging for about 10 minutes $(4000 \mathrm{r} / \mathrm{min})$. The supernatant in a volume of $0.1 \mathrm{~mL}$ was pipetted into $5 \mathrm{~mL}$ test tubes, and $3 \mathrm{~mL}$ of Coomassie Brilliant Blue G-250 dye reagent was added to the test tube, then the contents were mixed by vortexing. The absorbance at $595 \mathrm{~nm}$ was measured after 10-15 minutes. The standard curve was plotted between insulin content and corresponding absorbance. The $\mathrm{AE}$ and LC of the insulinloaded WSC-MPs were calculated according to (1). The amounts of insulin were determined in triplicate:

$$
\begin{aligned}
& \text { AE }(\%) \\
& =\frac{\text { Total amount of insulin }- \text { Free insulin in supernatant }}{\text { Total amount of insulin }} \\
& \quad \times 100, \\
& \text { LC }(\%) \\
& =\frac{\text { Total amount of insulin }- \text { Free insulin in supernatant }}{\text { Total weight of nanoparticles }} \\
& \quad \times 100 .
\end{aligned}
$$


2.7. In Vitro Release Studies. To establish the insulin release profile from insulin-loaded WSC-MPs at simulated gastric and intestinal $\mathrm{pH}$, insulin-loaded WSC-MPs were placed into test tubes containing $1.5 \mathrm{~mL}$ of $\mathrm{pH} 1.2$ and $\mathrm{pH} 7.4$ phosphate buffer at $37^{\circ} \mathrm{C}$, respectively. The concentrations of insulinloaded WSC-MPs in the release medium were adjusted in order to achieve sink conditions for insulin release. At appropriate time intervals of $0.5,2,4,6,8,10,12$, and $24 \mathrm{~h}$, $1 \mathrm{~mL}$ of samples removed and ultracentrifuged at $15,000 \times \mathrm{g}$ for 30 minutes, and $1 \mathrm{~mL}$ of the supernatants was replaced by fresh medium. The individual sample was centrifuged, and the amount of insulin in the supernatant was measured by the improved Bradford method. All release experiments were done triplicately, and the mean values were recorded. The insulin cumulative release was calculated according to

$$
\mathrm{CR}(\%)=\frac{C_{t} \times V+V_{i} \sum_{n=0}^{t-1} C_{i}}{W \times X} \times 100,
$$

where $C_{t}$ is the sample concentration at $T, V$ was the total volume of release medium, $V_{i}$ was the sampling volume at $T_{i}$, $C_{i}$ was the sample concentration at $T_{i}$ (both $V_{0}$ and $C_{0}$ were equal to zero), $W$ was the weight of lyophilized particles used for drug content determination, and $X$ was the drug content.

\section{Results and Discussion}

3.1. Preparation of WSC-MPs. Experiments were performed by using various WSC aqueous solutions and TPP concentration, and the optimal condition was selected to achieve microparticles by polyelectrolyte complexation. The results are presented in Table 1.

The zone of the opalescence corresponding to a suspension of WSC-MPs was associated with a formulation containing a final WSC aqueous solution concentration in the range $1.0-2.0 \mathrm{mg} / \mathrm{mL}$ and a final TPP concentration in the range $0.5-1.0 \mathrm{mg} / \mathrm{mL}$, respectively. These conditions were selected for preparation of the WSC-MPs to reach final theoretical WSC/TPP ratios of $6: 1-8: 1(\mathrm{w} / \mathrm{w})$.

\subsection{Preparation of Insulin-Loaded WSC-MPs}

3.2.1. Effect of Resolution Medium of Insulin. Table 2 represents the insulin association efficiency of the insulin-loaded WSC-MPs prepared by the ionotropic gelation in different resolution meda of insulin. Insulin was premixed with WSC aqueous solution prior to MPs' formation.

The results showed higher AE for insulin-loaded WSCMPs in $0.01 \mathrm{~mol} / \mathrm{L} \mathrm{HCl}$ than other resolution medium of insulin. This finding can probably be explained by the competition between insulin and TPP in the interaction with WSC. Insulin in $0.01 \mathrm{~mol} / \mathrm{L} \mathrm{HCl}$ with higher negative charge density resulted in prior formation of insulin-loaded WSC-MPs; the particles were formed spontaneously by the electrostatic interactions of positively charged WSC and negatively charged insulin. Compared to insulin, TPP is a much smaller molecule with higher negative charge density. It can dominate the interaction of insulin with positively charged polymers causing a reduction in the positive charge density of WSC.

\subsubsection{Optimization of Insulin-Loaded WSC-MPs. Many} studies have reported that the quantity of TPP in a given formulation has a significant effect on the insulin encapsulation and characteristic of CS-MPs $[5,6,8,20]$. Therefore, the optimal amount of TPP in formulation was investigated in detail.

Results of the investigations on the experimental conditions for the formation of insulin-loaded WSC-MPs could be obtained by varying the concentrations of TPP, WSC, and insulin. As concluded from the results given in Table 3, association efficiency of the insulin-loaded WSC-MPs was affected by the insulin concentration in $0.01 \mathrm{~mol} / \mathrm{L} \mathrm{HCl}$ solutions and the amount of insulin incorporated, with increasing amount of insulin leading to a slight decrease of association efficiency. The mechanism of insulin association to insulin-loaded WSC-MPs was mediated by an ionic interaction between both macromolecules. The electrostatic interactions between the acidic insulin groups and the amino groups of WSC might have played a role in association of insulin to the insulin-loaded WSC-MPs.

Formulation of insulin-loaded WSC-MPs contained a final WSC aqueous solution concentration of $1.5 \mathrm{mg} / \mathrm{mL}$ and a final TPP concentration of $1.0 \mathrm{mg} / \mathrm{mL}$, respectively. This condition was selected for preparation of the insulin-loaded WSC-MPs to reach final theoretical WSC/TPP ratios of $3: 1(\mathrm{w} / \mathrm{w})$. Insulin concentration of $0.5 \mathrm{mg} / \mathrm{mL}(0.01 \mathrm{~mol} / \mathrm{L}$ $\mathrm{HCl}$ ) could be loaded efficiently, and the relatively higher association efficiency and loading capacity of insulin-loaded WSC-MPs were $48.28 \pm 0.90 \%$ and $9.52 \pm 1.34 \%(n=3)$, respectively. However, the preparation of chitosan-insulin microparticles with about $30 \%$ of $\mathrm{AE}$ by a polyelectrolyte complexation method using insulin in $0.01 \mathrm{~mol} / \mathrm{L} \mathrm{HCl}$ was reported [23].

3.2.3. Effect of $p H$ of TPP. Table 4 represents the insulin association efficiency of the microparticles prepared by the polyelectrolyte complexation at different $\mathrm{pH}$ of TPP. WSC aqueous solution presented $-\mathrm{NH}_{3}{ }^{+}$sites. Tripolyphosphoric acid $\left(\mathrm{H}_{5} \mathrm{P}_{3} \mathrm{O}_{10}\right)$ is a weak polyprotic acid-like phosphoric acid, the $K_{\alpha}$ decrease from $K_{\alpha 1}$ to $K_{\alpha 2}$, and the decreased quantity is one to several orders. Due to this reason, sodium tripolyphosphate $\left(\mathrm{Na}_{5} \mathrm{P}_{3} \mathrm{O}_{10}\right)$ dissolved in water to dissociate both $\mathrm{OH}^{-}$and tripolyphosphoric ions in the TPP solution. In original TPP solution (basic, $\mathrm{pH}$ was not adjusted), the concentration of $\mathrm{P}_{3} \mathrm{O}_{10}{ }^{5-}$ and $\mathrm{HP}_{3} \mathrm{O}_{10}{ }^{4-}$ was high, but the concentration of $\mathrm{OH}^{-}$was also present. The $\mathrm{OH}^{-}$or tripolyphosphoric ions $\left(\mathrm{P}_{3} \mathrm{O}_{10}{ }^{5-}\right.$ and $\left.\mathrm{HP}_{3} \mathrm{O}_{10}{ }^{4-}\right)$ in this medium could competitively react ionically with the bind site $-\mathrm{NH}_{3}{ }^{+}$in chitosan by deprotonation and covalent bonds, respectively. The $\mathrm{pH}$ value of prepared chitosan $(0.01 \mathrm{M})$ or TPP aqueous solution $(0.01 \mathrm{M})$ was 4.0 and 9.7 , respectively. The TPP ions $\left(\mathrm{P}_{3} \mathrm{O}_{10}{ }^{5-}\right)$ competed with $\mathrm{OH}^{-}$ions to bind with $-\mathrm{NH}_{3}{ }^{+}$in chitosan, resulting in the decrease of binding sites for $\mathrm{OH}^{-}$ions and increased $\mathrm{pH}$. When titrating acetic acid ( $\mathrm{pH} 4.0$ ) with TPP ( $\mathrm{pH}$ 9.7), it was found that the $\mathrm{pH}$ of 
TABLE 1: Effect of water-soluble chitosan (WSC) and TPP concentrations on the formation of microparticles.

\begin{tabular}{|c|c|c|c|c|c|}
\hline \multirow{2}{*}{ WSC $(\mathrm{mg} / \mathrm{mL})$} & \multicolumn{5}{|c|}{$\mathrm{TPP}(\mathrm{mg} / \mathrm{mL})$} \\
\hline & 0.5 & 0.7 & 1.0 & 1.5 & 2.0 \\
\hline 0.8 & $\downarrow$ & $\downarrow$ & $\downarrow$ & $\downarrow$ & $\downarrow$ \\
\hline 1.0 & $\circ$ & $\downarrow$ & $\downarrow$ & $\downarrow$ & $\downarrow$ \\
\hline 1.5 & $\times$ & $\circ$ & $\downarrow$ & $\downarrow$ & $\downarrow$ \\
\hline 2.0 & $\times$ & $\circ$ & $\circ$ & $\downarrow$ & $\downarrow$ \\
\hline 3.0 & $x$ & $x$ & $x$ & $\downarrow$ & $\downarrow$ \\
\hline
\end{tabular}

$\times$ : clear solution; ॰: opalescent suspension (nanoparticles); $\downarrow$ : aggregation.

TABLE 2: Effect of resolution medium of insulin on association efficiency (AE) $(n=3)$.

\begin{tabular}{lc}
\hline Resolution medium & \% AE (mean \pm SD) \\
\hline $0.01 \mathrm{~mol} / \mathrm{LHCl}$ & $48.28 \pm 1.2$ \\
$0.01 \mathrm{~mol} / \mathrm{LNaOH}$ & $18.80 \pm 2.3$ \\
pH 7.4 phosphate buffer & $40.07 \pm 4.3$ \\
distilled water & $34.82 \pm 3.6$ \\
\hline
\end{tabular}

mixing increased quickly. This is due to the buffer ability of TPP ions in weak acid. However, the $\mathrm{pH}$ increase of the TPP ( $\mathrm{pH}$ 9.7)/chitosan ( $\mathrm{pH} 4.0$ ) mixing solution was significantly slower than the $\mathrm{pH}$ increase of TPP ( $\mathrm{pH}$ 9.7)/acid ( $\mathrm{pH} 4.0)$ mixture. This suggests that the TPP ion lost its buffer ability due to binding to the protonated chitosan. This hypothesis shows that the precipitations of complexes in this state were formed both by deprotonation and covalent bonds. By adjusting the $\mathrm{pH}$ value of TPP solution from 9.7 (initial) to 4.0, only $\mathrm{P}_{3} \mathrm{O}_{10}{ }^{5-}$ anions existed. The $\mathrm{pH}$ value of the TPP ( $\mathrm{pH} 4.0) /$ chitosan ( $\mathrm{pH}$ 4.0) mixture was kept almost constant with the increase of TPP/chitosan mixing ratio due to no $\mathrm{OH}^{-}$ions being released, but chitosan-TPP precipitation was also formed. This result shows that the precipitations of complexes were formed only by ionic-crosslinking between $-\mathrm{NH}_{3}{ }^{+}$and TPP ions [24]. So insulin-loaded WSC-MPs had higher association efficiency in the $\mathrm{pH} 4.0$ and $\mathrm{pH} 9.7$ of TPP solution.

3.3. Morphology of the Microparticles. According to the size distribution, a photograph and a TEM photograph are shown in Figures 1 and 2, respectively. Morphologically, the insulin-loaded WSC-MPs look round to oval in shape and have a relatively smooth surface. The average size of Insulinloaded WSC-MPs was $292 \mathrm{~nm}$.

3.4. Insulin Release Studies. The representative insulin release profile from insulin-loaded WSC-MPs in conditions, simulating the gastric and intestinal pH, is illustrated in Figure 3, as a suitable application of this method. The profile of drug release from nanoparticles can be affected by method of preparation and also by ionic interaction between the drug and addition of auxiliary ingredients. If the drug was loaded by incorporation method, the system would have a relatively small burst effect and then sustained release characteristics. When the drug was involved in interaction

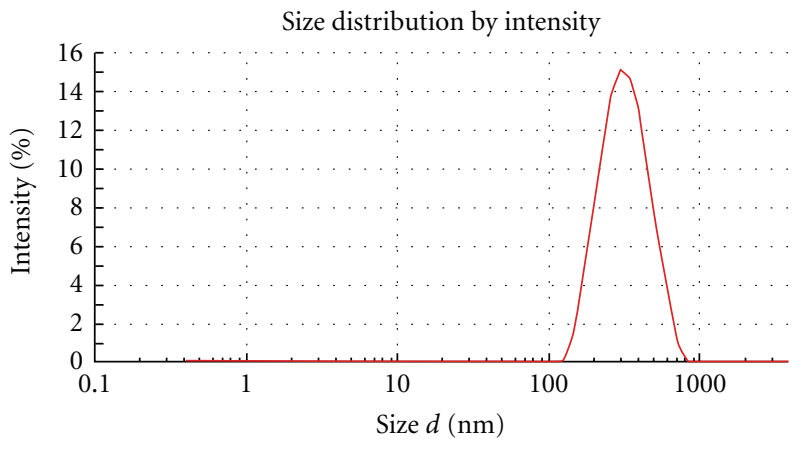

FIGURE 1: Size distribution of the insulin-loaded WSC-MPs made by the polyelectrolyte complexation.

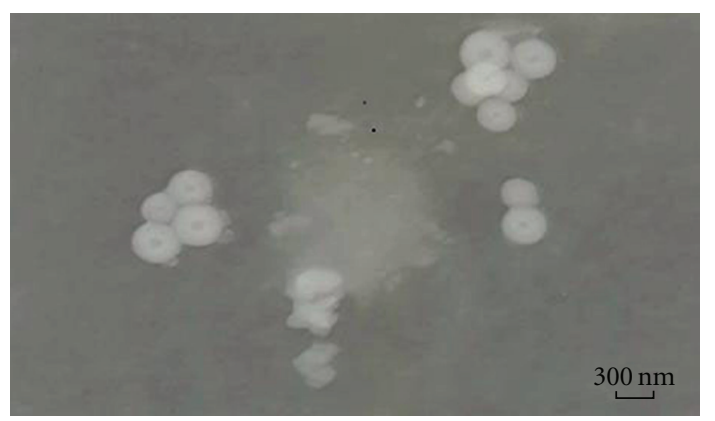

FIGURE 2: TEM photograph of the insulin-loaded WSC-MPs made by the polyelectrolyte complexation $(7 \mathrm{KV}, \times 1,600)$.

with auxiliary ingredients to form a less water-soluble complex, the drug release can be very slow with almost no burst effect. The release results showed good incorporation of insulin in polymers by ionic interaction between insulin and the polymers. Insulin release was characterized by no burst effect, in both media at $0.5 \mathrm{~h}(<40 \%)$. The preliminary insulin release test from insulin-loaded WSC-MPs in vitro proved that they had a sustained release form as shown in Figure 3. Ionic strength in release medium may affect significantly the release properties of insulin-loaded WSCMPs based on ionic gelation. The in vitro insulin release profiles obtained for each formulation showed three phases [25]: (1) a first initial burst release of $50 \%$ (in $2 \mathrm{~h}$ ), due to the drug desorption from the particles surface; (2) a plateau for the following $12 \mathrm{~h}$, resulting only from the diffusion of the drug dispersed in the polymer matrix; (3) a constant 
TABLE 3: The optimal conditions for the formulation of insulin-loaded WSC-MPs by orthogonal design $\left(\mathrm{L}_{9}\left(3^{4}\right)\right)(n=3)$.

\begin{tabular}{|c|c|c|c|c|c|}
\hline NO. & $\mathrm{pH}$ of WSC & $C_{\mathrm{WSC}}(\mathrm{mg} / \mathrm{mL})$ & $C_{\mathrm{INS}}(\mathrm{mg} / \mathrm{mL})$ & $C_{\mathrm{TPP}}(\mathrm{mg} / \mathrm{mL})$ & $\mathrm{AE}(\%)$ \\
\hline 1 & 3.8 & 1.0 & 0.5 & 0.7 & 32.01 \\
\hline 2 & 3.8 & 1.5 & 0.7 & 1.0 & 27.27 \\
\hline 3 & 3.8 & 2.0 & 1.0 & 1.5 & 26.32 \\
\hline 4 & 4.5 & 1.0 & 0.7 & 1.5 & 21.79 \\
\hline 5 & 4.5 & 1.5 & 1.0 & 0.7 & 31.24 \\
\hline 6 & 4.5 & 2.0 & 0.5 & 1.0 & 44.56 \\
\hline 7 & 5.0 & 1.0 & 1.0 & 1.0 & 34.91 \\
\hline 8 & 5.0 & 1.5 & 0.5 & 1.5 & 27.65 \\
\hline 9 & 5.0 & 2.0 & 0.7 & 0.7 & 15.95 \\
\hline
\end{tabular}

TABLE 4: Effect of $\mathrm{pH}$ of TPP on association efficiency (AE) $(n=3)$.

\begin{tabular}{lc}
\hline $\mathrm{pH}$ of TPP & \% AE $($ mean $\pm \mathrm{SD})$ \\
\hline $0.01 \mathrm{~mol} / \mathrm{LHCl}$ & $18.44 \pm 5.4$ \\
3.0 & $33.35 \pm 4.1$ \\
4.0 & $46.26 \pm 2.6$ \\
5.0 & $29.75 \pm 3.2$ \\
7.0 & $32.39 \pm 2.8$ \\
9.7 (distilled water) & $48.31 \pm 1.6$ \\
\hline
\end{tabular}

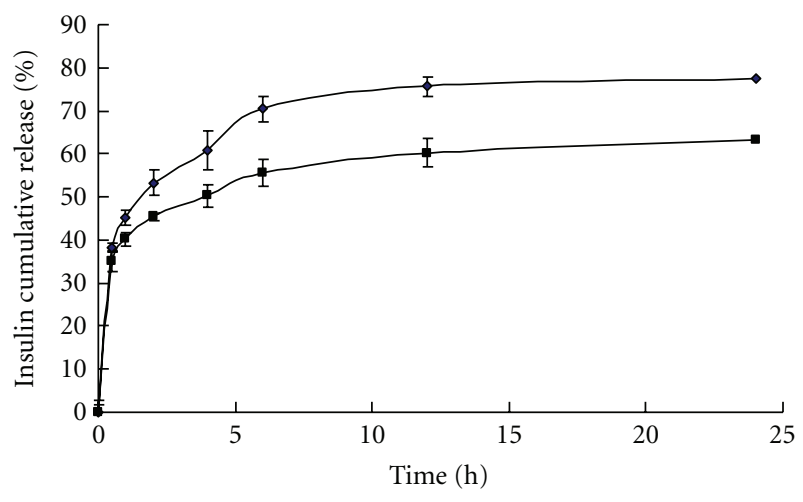

FIGURE 3: Insulin release from WSC-MPs at gastric $\mathrm{pH} 1.2(\checkmark)$ and phosphate buffer, simulating intestinal pH $7.4(\mathbf{\square})(n=3)$.

sustained release of the remaining drug, resulting from the diffusion of the insulin through the polymer wall as well as its erosion.

\section{Conclusion}

In summary, the results suggested that, while the polyelectrolyte complexation is easy to be used for the production of insulin-loaded WSC-MPs, the insulin in $0.01 \mathrm{~mol} / \mathrm{L} \mathrm{HCl}$ seems to be more suitable for future studies. The insulinloaded WSC-MPs obtained by the polyelectrolyte complexation method had higher insulin association efficiency and had higher association efficiency in $\mathrm{pH} 4.0$ and $\mathrm{pH}$ 9.7 of TPP solution. These insulin-loaded WSC-MPs have narrow size distributions look round to oval in shape, and have a relatively smooth surface. The in vitro release profiles of insulin from insulin-loaded WSC-MPs showed relatively small initial burst release followed by a slowly sustained release phase. Quantification of insulin released from insulin-loaded WSC-MPs by the improved Bradford method supported the application of these insulin-loaded WSC-MPs as oral delivery systems for insulin. Present investigations are being performed regarding the assessment of insulin in vivo oral bioactivity.

\section{Acknowledgments}

This project was financially supported by Science and Technology Planning Project of Guangdong Province, China (nos. 2009B020313006 and 2010B090400467) Science and Technology Planning Project of Zhongshan, China (no. 2009H017), and Foundation for University Key Teacher of Guangdong Pharmaceutical University, China. The authors thank the teachers of the Central Laboratory and Life Science and Bio-Pharmaceutical College of Guangdong Pharmaceutical University who provided equipment and guidance for the experiments.

\section{References}

[1] D. R. Owens, B. Zinman, and G. Bolli, "Alternative routes of insulin delivery," Diabetic Medicine, vol. 20, no. 11, pp. 886898, 2003.

[2] R. I. Mahato, A. S. Narang, L. Thoma, and D. D. Miller, "Emerging trends in oral delivery of peptide and protein drugs," Critical Reviews in Therapeutic Drug Carrier Systems, vol. 20, no. 2-3, pp. 153-214, 2003.

[3] J. H. Hamman, G. M. Enslin, and A. F. Kotzé, "Oral delivery of peptide drugs: barriers and developments," BioDrugs, vol. 19, no. 3, pp. 165-177, 2005.

[4] M. Morishita and N. A. Peppas, "Is the oral route possible for peptide and protein drug delivery?” Drug Discovery Today, vol. 11, no. 19-20, pp. 905-910, 2006.

[5] S. Sajeesh and C. P. Sharma, "Cyclodextrin-insulin complex encapsulated polymethacrylic acid based nanoparticles for oral insulin delivery," International Journal of Pharmaceutics, vol. 325, no. 1-2, pp. 147-154, 2006.

[6] A. Bayat, F. A. Dorkoosh, A. R. Dehpour et al., "Nanoparticles of quaternized chitosan derivatives as a carrier for colon delivery of insulin: ex vivo and in vivo studies," International Journal of Pharmaceutics, vol. 356, no. 1-2, pp. 259-266, 2008. 
[7] C. Damgé, P. Maincent, and N. Ubrich, "Oral delivery of insulin associated to polymeric nanoparticles in diabetic rats," Journal of Controlled Release, vol. 117, no. 2, pp. 163-170, 2007.

[8] B. Sarmento, A. Ribeiro, F. Veiga, and D. Ferreira, "Development and characterization of new insulin containing polysaccharide nanoparticles," Colloids and Surfaces B, vol. 53, no. 2, pp. 193-202, 2006.

[9] V. J. Mohanraj and Y. Chen, "Nanoparticles-a review," Tropical Journal of Pharmaceutical Research, vol. 5, no. 3, pp. 561-473, 2006.

[10] R. Yang, W. S. Shim, F. D. Cui et al., "Enhanced electrostatic interaction between chitosan-modified PLGA nanoparticle and tumor," International Journal of Pharmaceutics, vol. 371, no. 1-2, pp. 142-147, 2009.

[11] C. Prego, D. Torres, E. Fernandez-Megia, R. Novoa-Carballal, E. Quiñoá, and M. J. Alonso, "Chitosan-PEG nanocapsules as new carriers for oral peptide delivery: effect of chitosan pegylation degree," Journal of Controlled Release, vol. 111, no. 3, pp. 299-308, 2006.

[12] A. Bayat, B. Larijani, S. Ahmadian, H. E. Junginger, and M. Rafiee-Tehrani, "Preparation and characterization of insulin nanoparticles using chitosan and its quaternized derivatives," Nanomedicine, vol. 4, no. 2, pp. 115-120, 2008.

[13] L. X. Liu, C. N. Song, L. P. Song, H. L. Zhang, X. Dong, and X. G. Leng, "Effects of alkylated-chitosan-DNA nanoparticles on the function of macrophages," Journal of Materials Science, vol. 20, no. 4, pp. 943-948, 2009.

[14] B. Sayin, S. Somavarapu, X. W. Li et al., "Mono-Ncarboxymethyl chitosan (MCC) and N-trimethyl chitosan (TMC) nanoparticles for non-invasive vaccine delivery," International Journal of Pharmaceutics, vol. 363, no. 1-2, pp. 139148, 2008.

[15] E. Ieva, A. Trapani, N. Cioffi, N. Ditaranto, A. Monopoli, and L. Sabbatini, "Analytical characterization of chitosan nanoparticles for peptide drug delivery applications," Analytical and Bioanalytical Chemistry, vol. 393, no. 1, pp. 207-215, 2009.

[16] R. Wang, B. Xia, B. J. Li, S. L. Peng, L. S. Ding, and S. Zhang, "Semi-permeable nanocapsules of konjac glucomannanchitosan for enzyme immobilization," International Journal of Pharmaceutics, vol. 364, no. 1, pp. 102-107, 2008.

[17] L. Zhu, J. Ma, N. Jia, Y. Zhao, and H. Shen, "Chitosan-coated magnetic nanoparticles as carriers of 5-Fluorouracil: preparation, characterization and cytotoxicity studies," Colloids and Surfaces B, vol. 68, no. 1, pp. 1-6, 2009.

[18] Y. Xu and Y. Du, "Effect of molecular structure of chitosan on protein delivery properties of chitosan nanoparticles," International Journal of Pharmaceutics, vol. 250, no. 1, pp. 215226, 2003.

[19] S. Zhu, F. Qian, Y. Zhang, C. Tang, and C. Yin, "Synthesis and characterization of PEG modified N-trimethylaminoethylmethacrylate chitosan nanoparticles," European Polymer Journal, vol. 43, no. 6, pp. 2244-2253, 2007.

[20] R. Fernández-Urrusuno, P. Calvo, C. Remuñán-López, J. L. Vila-Jato, and M. J. Alonso, "Enhancement of nasal absorption of insulin using chitosan nanoparticles," Pharmaceutical Research, vol. 16, no. 10, pp. 1576-1581, 1999.

[21] P. Calvo, C. Remuñán-López, J. L. Vila-Jato, and M. J. Alonso, "Novel hydrophilic chitosan-polyethylene oxide nanoparticles as protein carriers," Journal of Applied Polymer Science, vol. 63, no. 1, pp. 125-132, 1997.

[22] Z. Q. Su, S. H. Wu, H. L. Zhang, and Y. F. Feng, “Development and validation of an improved Bradford method for determination of insulin from chitosan nanoparticulate systems," Pharmaceutical Biology, vol. 48, no. 9, pp. 966-973, 2010.
[23] Z. Ma, T. M. Lim, and L. Y. Lim, "Pharmacological activity of peroral chitosan-insulin nanoparticles in diabetic rats," International Journal of Pharmaceutics, vol. 293, no. 1-2, pp. 271-280, 2005.

[24] M. I. Fwu-Long, S. S. Shyu, S. T. Lee, and T. B. I. Wong, "Kinetic study of chitosan-tripolyphosphate complex reaction and acid-resistive properties of the chitosan-tripolyphosphate gel beads prepared by in-liquid curing method," Journal of Polymer Science, Part B, vol. 37, no. 14, pp. 1551-1564, 1999.

[25] A. Lamprecht, N. Ubrich, M. H. Pérez, C. M. Lehr, M. Hoffman, and P. Maincent, "Influences of process parameters on nanoparticle preparation performed by a double emulsion pressure homogenization technique," International Journal of Pharmaceutics, vol. 196, no. 2, pp. 177-182, 2000. 

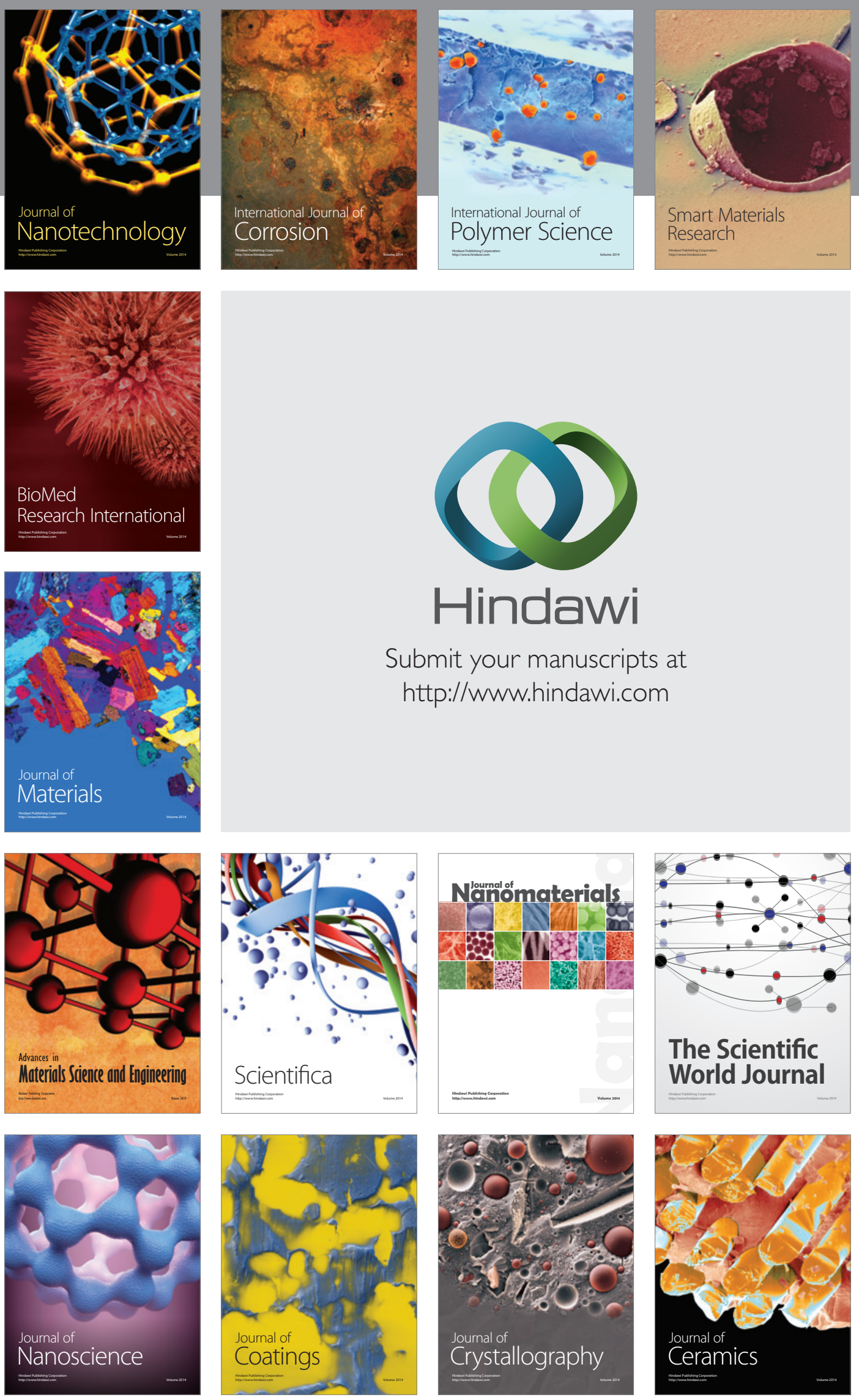

The Scientific World Journal

Submit your manuscripts at

http://www.hindawi.com

\section{World Journal}

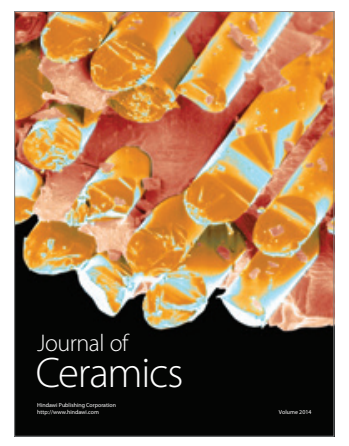

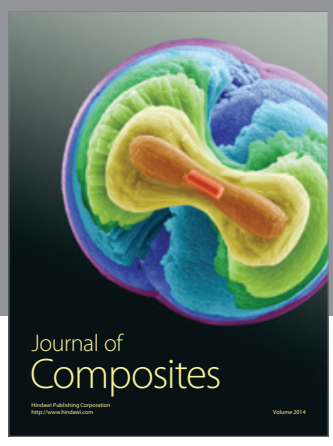
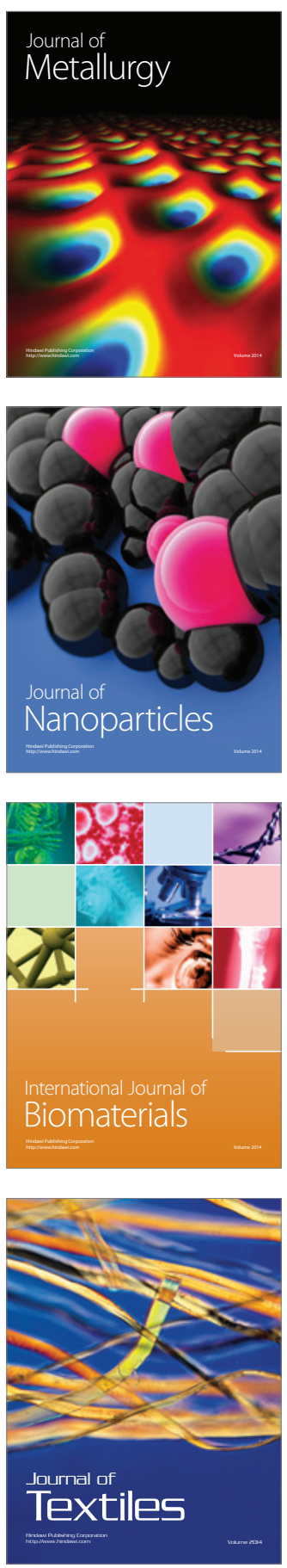